\title{
Urinary alkylresorcinol metabolites as a biomarker of dietary wholegrain intake and of compliance in a randomised dietary intervention trial: results from the CRESSIDA Study
}

\author{
J. Cuff, T. A. B. Sanders, D. P. Reidlinger, W. L. Hall, R. Gray and J. Darzi \\ Diabetes and Nutritional Sciences Division, King's College London, London SE1 9NH
}

Alkylresorcinols (AR) are a family of phenolic lipids found in the bran fraction of whole grain (WG) wheat and rye, and absent in refined cereals. The AR metabolites, 3,5-dihydroxybenzoic acid (DHBA) and 3-(3,5-dihydroxyphenyl)-propanoic acid (DHPPA) have been proposed as a biomarker of WG intake, and are quantifiable in both plasma and urine ${ }^{(1)}$. However, this is yet to be confirmed in a low (predominantly wheat) WG consuming population, such as in the UK. The present study evaluated the validity and reproducibility of 24-h urinary DHBA, DHPPA and $\mathrm{AR}_{\mathrm{TOT}}$ (DHBA + DHPPA) excretion, as a biomarker of WG intake, in healthy UK adults, using samples from the CRESSIDA study (ISRCTN92382106).

DHBA and DHPPA excretion was quantified by a modified GC-MS method ${ }^{(2)}$, in 24-h urine samples collected from 162 healthy men and women, aged 40-70 y. Samples were collected twice at baseline and once at endpoint, following a 12-wk dietary intervention, in which participants were randomised to follow a diet high in refined cereals $(\mathrm{CON})$ or a diet close to dietary guidelines, with an increased content of WG (DG). Dietary intake was assessed by 4-d food diary. Reproducibility was assessed by intra class correlation coefficient (ICC) between duplicate baseline urine collections. Validity was assessed by Spearman's rank test correlations between baseline 24-h urinary AR metabolite excretion and estimated intake of WG, total fibre and cereal fibre. Completeness of urine collection was assessed using the para-amino benzoic acid recovery method (ADJ), a ratio to creatinine (AR:CR) and without adjustment (UNADJ). All data was log transformed prior to statistical analysis.

Moderate to high reproducibility was observed for DHBA $(\mathrm{ICC}=0.552-0.620)$ which was greater than that observed for either DHPPA ( $\mathrm{ICC}=0.427-0.527)$ or $\mathrm{AR}_{\mathrm{TOT}}(\mathrm{ICC}=0.488-0.583)$ and higher than that observed in previously published studies ${ }^{(3)}$. In terms of validity, significant and moderate associations between 24-h urinary excretion and estimated total fibre, cereal fibre and WG intake were found, with the strongest associations for DHBA. Following a 12-week intervention, endpoint AR metabolite excretion was significantly higher following DG vs. CON. For all AR metabolites, the strongest associations with estimated dietary intakes were for AR:CR, followed by UNADJ, with the weakest associations with ADJ recoveries.

\begin{tabular}{|c|c|c|c|c|c|c|c|}
\hline & Repro & & Validi & & Treatment ef & & \\
\hline & $\mathrm{ICC}$ & $P$ value & $\mathrm{R}_{\mathrm{S}}$ & $P$ value & $\%$ difference & $95 \% \mathrm{CI}$ & $P$ value \\
\hline DHBA & 0.620 & $<0.0001$ & $0 \cdot 381$ & $<0 \cdot 0001$ & 36 & 13,58 & 0.002 \\
\hline DHPPA & $0 \cdot 527$ & $<0.0001$ & $0 \cdot 315$ & $<0.0001$ & 33 & 6,60 & $0 \cdot 017$ \\
\hline $\mathrm{AR}_{\mathrm{TOT}}$ & 0.583 & $<0.0001$ & $0 \cdot 355$ & $<0.0001$ & 35 & 12,58 & 0.003 \\
\hline
\end{tabular}

${ }^{1}$ All data presented for log transformed AR:CR urinary excretion, ${ }^{2}$ Correlation assessed by Spearman's Rank Test in comparison to estimated WG intake, ${ }^{3}$ Difference between DG and CON treatments at endpoint assessed by ANCOVA adjusted for baseline AR metabolite excretion, gender, BMI and age.

These results indicate that all AR metabolites are valid and reproducible biomarkers of WG intake in the adult UK population, where wheat is the primary dietary source of WG. The strongest associations with estimated intake were found for DHBA. By contrast, in Nordic populations, DHPPA was the main predictor of WG intake ${ }^{(3,4)}$ probably because rye is consumed in higher amounts as opposed to wheat in the present study. These data suggest total urinary AR adjusted for creatinine may be a useful biomarker of wholegrain intake.

Funding was from the Food Standards Agency and the Department of Health. Currently the project is supported by Public Health England, which is responsible for nutrition advice since April 2013.

1. Ross AB. (2012) J Nutr Metab 2012, 1-12

2. Marklund M, Landberg R, Åman P et al. (2010) J Chromatogr B, 878, 888-894.

3. Marklund M, Landberg R, Andersson A et al. (2013) Br J Nutr 109, 129-36.

4. Söderholm PP, Lundin JE, Koskela AH et al. (2011) Br J Nutr 106, 1040-4. 